\title{
ADAPTIVE CONTROL AND SYNCHRONIZATION OF SPROTT J SYSTEM WITH ESTIMATION OF FULLY UNKNOWN PARAMETERS*
}

\author{
Mitul Islam \\ 203, B.T. Road, Indian Statistical Institute, \\ Kolkata 700108, India, \\ e-mail: mitul.islam@gmail.com \\ NuRUL ISLAM \\ Ramakrishna Mission College (Autonomous), Narendrapur, \\ Kolkata 700103, India, \\ e-mail:dr.i.nurul@gmail.com \\ Svetoslav NikOlov \\ Institute of Mechanics, Bulgarian Academy of Sciences, \\ Acad. G. Bonchev St., Bl. 4, 1113 Sofia, Bulgaria, \\ University of Transport, 158, G. Milev St., 1574 Sofia, Bulgaria, \\ e-mail:s.nikolov@imbm.bas.bg
}

[Received 16 April 2015. Accepted 15 June 2015]

\begin{abstract}
This communication develops an adaptive scheme for control and synchronization of Sprott J system with fully unknown parameters. The scheme provides an elegant strategy of designing estimators for identification of the unknown parameters of the underlying dynamical system. Adaptive control and update laws are proposed to globally stabilize the chaotic Sprott J system. A pair of identical Sprott J systems with unknown parameters are globally synchronized with the help of adaptive control and parameter update laws. The results are established using LaSalle invariance principle, which lays down weaker restrictions on the derivatives of the Lyapunov function, and producing more general results. All the results obtained in the paper are global in nature. Numerical simulations are performed to illustrate the validity and effectiveness of the proposed adaptive control and synchronization scheme in the context of the Sprott J system. The parameter identification capability of the scheme is also explored.
\end{abstract}

\footnotetext{
${ }^{*}$ Corresponding author e-mail: s.nikolov@imbm.bas.bg

Svetoslav Nikolov is funded by the grant No 1616/22.04.2014 of the University of Transport "T. Kableshkov" Sofia, Bulgaria.
} 
KEY wORDS: Sprott J system, adaptive control, adaptive synchronization; LaSalle invariance principle; Lyapunov stability theorem.

\section{Introduction}

Chaos and non-linear dynamics [1] are presently active fields of interdisciplinary research, attracting the attention of researchers on account of its wide applicability in the physical world. One of the key problems in study of chaotic systems is the control of chaos. Its importance is on account of its application in diverse fields like cryptography, steganography, engineering design, non-linear control systems, and so on. A host of other chaotic systems have been discovered since Edward Lorenz's [2] discovery in 1963. Fairly recently, Sprott has proposed a set of chaotic dynamical systems purely from a mathematical standpoint; Sprott J system [3] being one of them. These particularly simple polynomial dynamical systems exhibit rich dynamical behaviour and have been in the center stage of studies for quite some time [4-8].

The chaos control problem is concerned with stabilization of the chaotic attractor to a periodic orbit or a stable equilibrium point. The pioneering works of Ott et al. [9-11] are the most important works in this direction. It was promptly followed by application of various engineering control techniques like feedback control [12], adaptive control [13], etc. for achieving this objective. The other popular method of chaos control is chaos synchronization. In the context of synchronizing two identical oscillators, the most prevalent method is to couple the systems suitably, so that they asymptotically follow the same path on the attractor. The master-slave, or drive-response formalism is commonly used for synchronization problems involving identical oscillators [14, 15].

A very promising chaos control strategy, that is lately being explored is the 'adaptive' approach [16-19]. Adaptive control [20, 21] and synchronization $[14,22,23]$ involves design of controllers, based on principle of engineering adaptive controllers. As opposed to conventional controllers, they are capable of adjusting themselves with changes in the original system, that they are supposed to control. This flexibility enhances their effectiveness. On this account, adaptive controllers are more appropriate for controlling chaos as opposed to many other commonly used control mechanisms like linear and non-linear feedback, tracking control, etc. Adaptive synchronization employs similar self-adjusting controllers to achieve complete synchronization between two similar or different chaotic systems. In this paper, methods for adaptive control and synchronization of Sprott J system are developed.

In real world applications, the original system, that is, the system which has to be controlled, is often inaccessible and hence the parameters guiding the 
system are either partially or completely unknown. Such errors might prove to be fatal due to the sensitive dependence of chaotic systems on initial conditions. Efficient controller designs should take this vital aspect into consideration. The controller should be robust enough to adjust itself accordingly in case of errors in measurement of system parameters. This aspect of controller design countered in this paper by using estimators that approximate the system parameters effectively.

Our paper is organized as follows. In Section 2, Sprott J system [3] is introduced and its stability behaviour is analyzed. Following this, in Section 3 adaptive controllers are designed for fully unknown system parameters. Parameter update laws are proposed which guarantee precise estimation of those parameters, besides controlling the chaotic Sprott J system. Adaptive synchronization of the system is taken up in Section 4. The synchronizing oscillators considered in the paper possess the same structural form but their parameters are fully unknown. The result of globally stable synchronization has been established with much weaker restrictions on the derivatives of the Lyapunov function in contrast to many traditional papers in the literature. This has been achieved by using LaSalle invariance principle in place of the more restrictive Lyapunov stability theorems. All the results obtained in this paper are global in nature. The Section 5 computationally verifies our claims and explains the results of the numerical simulation work, as here we also discuss our findings. Finally, in Section 6 we summarize our results.

\section{Sprott J system}

\subsection{Description of the system}

The Sprott J system is described by the system of equations,

$$
\begin{aligned}
& \dot{x}=\alpha z, \\
& \dot{y}=-\beta y+z, \\
& \dot{z}=-x+y+y^{2},
\end{aligned}
$$

where, $\alpha>0$ and $\beta>0$ are system parameters. It is easy to see that the system (1) has only one critical (fixed) point $E_{0}=(0,0,0)$.

\subsection{Stability behaviour at the critical point}

Theorem 1. The critical point $E_{0}=(0,0,0)$ of the system (1) is always unstable.

Proof. The Jacobian matrix of the system (1) about the critical point 
$E_{0}=(0,0,0)$ is:

$$
J_{0}=\left(\begin{array}{ccc}
0 & 0 & \alpha \\
0 & -\beta & 1 \\
-1 & 1 & 0
\end{array}\right)
$$

Hence, the characteristic equation possesses the form:

$$
\lambda^{3}+\beta \lambda^{2}-(\alpha-1) \lambda+\alpha \beta=0,
$$

where $a_{1}=\beta, a_{2}=\alpha-1$ and $a_{3}=\alpha \beta$. According to the Routh-Hurwitz criteria the critical point $E_{0}=(0,0,0)$ of the system (1) will be stable if $a_{1}>0, a_{2}>0$, $a_{3}>0$, and $a_{1} a_{2}-a_{3}>0$.

Clearly, $a_{1}=\beta>0, a_{3}=\alpha \beta>0$ and $a_{2}=\alpha-1>0$, that is:

$$
\alpha>1 .
$$

Also, $a_{1} a_{2}-a_{3}>0$ gives $\beta(\alpha-1)>\alpha \beta$ that is, $\alpha-1>\alpha$ which is not possible.

So, the critical point will be always unstable. Since the critical point of the system (1) is unstable, the control problem takes place.

Remark 1. According to [3], the system (1) is chaotic for $\alpha=2$ and $\beta=2$. Clearly, the relation (2) is satisfied by these values of $\alpha$ and $\beta$. Hence the equilibrium $E_{0}$ of the system is unstable when the parameters are in the chaotic regime.

\section{Adaptive control of Sprott J system with fully unknown parameters}

Consider the system (1) with fully unknown parameters $\alpha$ and $\beta$. As these system parameters are not available for measurement, any controller that utilizes these parameters must be accompanied by suitable estimators for the respective parameters. So, a general control problem for system (1) with fully uncertain parameters may be formulated as:

Determine control inputs $u_{1}, u_{2}, u_{3}$ such that:

$$
\left(\begin{array}{c}
\dot{x} \\
\dot{y} \\
\dot{z}
\end{array}\right)=\left(\begin{array}{c}
\alpha z \\
-\beta y+z \\
-x+y+y^{2}
\end{array}\right)+\left(\begin{array}{l}
u_{1} \\
u_{2} \\
u_{3}
\end{array}\right),
$$

is globally asymptotically stable at the equilibrium point $E_{0}$. 
A simple and effective choice of $u_{1}, u_{2}$ and $u_{3}$ will be proposed in the following theorem. Since these functions might involve system parameters, they have to be designed in terms of the estimated values of the unknown parameters of the system. Let the estimators for $\alpha$ and $\beta$ be $\hat{\alpha}$ and $\hat{\beta}$, respectively. The following theorem gives the adaptive control laws for stabilizing the system at $E_{0}$.

Theorem 2. The system (3) is globally asymptotically stabilized at $E_{0}$ by the control input:

$$
\begin{aligned}
& u_{1}=-\hat{\alpha} z-k_{1} x, \\
& u_{2}=\hat{\beta} y-y z-k_{2} y, \\
& u_{3}=-k_{3} z,
\end{aligned}
$$

and the parameter estimator update laws:

$$
\begin{aligned}
& \dot{\hat{\alpha}}=x z-k_{4}(\hat{\alpha}-\alpha), \\
& \dot{\hat{\beta}}=-y^{2}-k_{5}(\hat{\beta}-\beta),
\end{aligned}
$$

for positive real constants $k_{1}, \ldots, k_{5}$ provided that:

$$
k_{1}\left(k_{2} k_{3}-1\right)>\frac{k_{2}}{4}
$$

where $\hat{\alpha}$ and $\hat{\beta}$ are estimators for the unknown system parameters $\alpha$ and $\beta$, respectively.

Moreover, if all the feedback gains are chosen to be equal, that is, $k_{i}=k$ for $i=1,2, \ldots, 5$, the controller can stabilize the system (3) at $E_{0}$ provided:

$$
k>\frac{\sqrt{5}}{2} \text {. }
$$

Proof. Substituting the adaptive control laws, as stated above, in the controlled system (3), one obtains:

$$
\begin{aligned}
& \dot{x}=\alpha z+\hat{\alpha} x \\
& \dot{y}=-\beta y+z+\hat{\beta}) y-y z, \\
& \dot{z}=-x+y+y^{2}-k z .
\end{aligned}
$$

Define the Lyapunov function $V: \mathbb{R}^{5} \rightarrow \mathbb{R}$ by:

$$
V=\frac{1}{2}\left[x^{2}+y^{2}+z^{2}+(\hat{\alpha}-\alpha)^{2}+(\hat{\beta}-\beta)^{2}\right] .
$$


Then,

$$
\dot{V}=-X^{T} A X
$$

where:

$$
X=(x, y, z, \hat{\alpha}-\alpha, \hat{\beta}-\beta)^{T},
$$

and

$$
A=\left(\begin{array}{ccccc}
k_{1} & 0 & 1 / 2 & 0 & 0 \\
0 & k_{2} & -1 & 0 & 0 \\
1 / 2 & -1 & k_{3} & 0 & 0 \\
0 & 0 & 0 & k_{4} & 0 \\
0 & 0 & 0 & 0 & k_{5}
\end{array}\right)
$$

Now, $\dot{V}$ is negative definite if and only if $A$ is positive definite. Clearly, $A$ is positive definite if and only if:

$$
k_{1}\left(k_{2} k_{3}-1\right)>\frac{k_{2}}{4}
$$

since $k_{i}>0, i=1,2, \ldots, 5$. Hence, the theorem follows by an easy application of Lyapunov stability theorems.

The second result is easy to observe by putting $k_{i}=k$ in the above relation.

\section{Adaptive synchronization with estimation of fully unknown} parameters

Consider a pair of systems with identical dynamics, coupled using the master-slave formalism:

$$
\begin{aligned}
\dot{X} & =f(X, \theta), \\
\dot{\hat{X}} & =f(\hat{X}, \hat{\theta})+U,
\end{aligned}
$$

where $X, \hat{X} \in \mathbb{R}^{n}$ are the states of the system and $\theta, \hat{\theta} \in \mathbb{R}^{m}$ are the parameters of the system and $U$ is the coupling function. In the standard unidirectional coupling scenarios, it is generally assumed that the parameters of the master system are known exactly a priori and hence, $\hat{\theta}=\theta$. In this paper, we consider the problem, where the master system parameters are unavailable. Hence, the synchronization scheme must involve parameter estimators, that will provide reasonable approximation of the system parameters. In other words, the dynamics of $\hat{\theta}$ has to be modelled such that they converge to the master system parameter $\theta$, at least asymptotically. Moreover, the convergence should 
ideally be globally stable, in order to ensure, that the scheme is effective and independent of the values of the master system's parameters.

Let $(X, \hat{X})$ be a master-slave pair of Sprott J dynamical systems, i.e.:

$$
\begin{aligned}
& \dot{x}=\alpha z, \quad \dot{\hat{x}}=\hat{\alpha} \hat{z}+U_{1}, \\
& X: \dot{y}=-\beta y+z, \quad \hat{X}: \dot{\hat{y}}=-\hat{\beta} \hat{y}+\hat{z}+U_{2} \text {, } \\
& \dot{z}=-x+y+y^{2}, \quad \dot{\hat{z}}=-\hat{x}+\hat{y}+\hat{y}^{2}+U_{3} \text {. }
\end{aligned}
$$

The aim is:

To determine coupling function $U$ and the parameter estimator evolution laws such that:

$$
\lim _{t \rightarrow \infty}\|X-\hat{X}\|=0
$$

For this coupled system (9), let $X=(x, y, z)^{T}$ and $\hat{X}=(\hat{x}, \hat{y}, \hat{z})^{T}$. Let us define $e_{x}=\hat{x}-x, e_{y}=\hat{y}-y, e_{z}=\hat{z}-z, e_{\alpha}=\hat{\alpha}-\alpha, e_{\beta}=\hat{\beta}-\beta$. Here, $\hat{\alpha}$ and $\hat{\beta}$ are estimators of the unknown system parameters $\alpha$ and $\beta$.

Theorem 3. A coupled pair of identical systems of the form (9) with completely unknown parameters $\alpha$ and $\beta$ is globally asymptotically synchronized under the coupling $U=\left(U_{1}, U_{2}, U_{3}\right)$ with:

$$
\begin{aligned}
& U_{1}=-\hat{\alpha} e_{z}-k_{1} e_{x}, \\
& U_{2}=\hat{\beta} e_{y}-2 e_{z}-k_{2} e_{y}, \\
& U_{3}=e_{x}-\hat{y}^{2}+y^{2}-k_{3} e_{z},
\end{aligned}
$$

and estimator evolution laws:

$$
\begin{aligned}
& \dot{\hat{\alpha}}=-z e_{x}, \\
& \dot{\hat{\beta}}=y e_{y},
\end{aligned}
$$

for any choice of positive real constants $k_{1}, k_{2}$ and $k_{3}$. theorem:

Proof. Using the coupling functions and the update laws as in the

$$
\begin{aligned}
& \dot{e}_{x}=-k_{1} e_{x}+e_{\alpha} z, \\
& \dot{e}_{y}=-k_{2} e_{y}-e_{\beta} y-e_{z}, \\
& \dot{e}_{z}=e_{y}-k_{3} e_{z}, \\
& \dot{\hat{\alpha}}=-z e_{x}, \\
& \dot{\hat{\beta}}=y e_{y} .
\end{aligned}
$$

Define the Lyapunov function:

$$
V=\frac{1}{2}\left(e_{x}^{2}+e_{y}^{2}+e_{z}^{2}+e_{\alpha}^{2}+e_{\beta}^{2}\right)
$$


Then:

$$
\dot{V}=-k_{1} e_{x}^{2}-k_{2} e_{y}^{2}-k_{3} e_{z}^{2}
$$

Let us consider the set:

$$
\begin{aligned}
S & =\{X: \dot{V}(X)=0\} \\
& =\left\{\left(0,0,0, r_{1}, r_{2}\right): r_{1}, r_{2} \in \mathbb{R}\right\}
\end{aligned}
$$

Claim. The only invariant trajectory of (12) in $S$ is the constant trajectory $\gamma \equiv 0$.

Proof of Claim. Let $\gamma: I \rightarrow S \subset \mathbb{R}^{5}$ be an invariant trajectory of (12) in $\mathrm{S}$ (I is an interval containing 0 , where the solution of (12) is defined), that is, $\gamma$ satisfies (12), $\gamma(0) \in S$ and $\gamma(I) \subset S$. As $\gamma(t) \in S$ for all $t \in I$, letting $\gamma(t)=\left(\gamma_{1}(t), \ldots, \gamma_{5}(t)\right)$ we have:

$$
\gamma_{1}(t)=\gamma_{2}(t)=\gamma_{3}(t)=0,
$$

and:

$$
\gamma_{4}(t)=r_{1}^{*}(t) \text { and } \gamma_{5}(t)=r_{2}^{*}(t) .
$$

Using (12) and (13), it is easy to observe, that:

$$
\dot{\gamma}_{4}(t)=\dot{\gamma}_{5}(t)=0 \text {, for all } t \in I,
$$

that is, both $\gamma_{4}$ and $\gamma_{5}$ are constants. Let $\gamma_{4}(t)=r_{1}$ and $\gamma_{5}(t)=r_{2}$ for all $t \in I$, for fixed real numbers $r_{1}$ and $r_{2}$. It remains to show that $r_{1}=r_{2}=0$.

Suppose $r_{1} \neq 0$. Then, using (12), we have $\dot{\gamma}_{1}(t)=z(t) r_{1}$. From the behaviour of system (1), we know that $z \triangleq 0$. Let $t_{0} \in I$, such that $z\left(t_{0}\right) \neq 0$. Then, $\dot{\gamma}_{1}\left(t_{0}\right)=z\left(t_{0}\right) r_{1} \neq 0$, contradicting the constancy of $\gamma_{1}(t)$ as observed in (13). Thus, we must have $r_{1}=0$.

Similarly, it can be proved that $r_{2}=0$. Hence, we have proved that any invariant curve in $\mathrm{S}$ is the constant curve $\gamma \equiv 0$.

Thus, $V$ is a positive definite, radially unbounded function with negative semidefinite derivative. Further, the set $S=\{x: \dot{V}(x)=0\}$ has the constant trajectory at origin, as the only invariant trajectory. Thus, by LaSalle invariance principle, the origin is a globally asymptotically stable equilibrium of the system, given by (12). The global stability of this error system ensures globally stable synchronization between $X$ and $\hat{X}$ along with convergence of the estimators to the desired value, that is:

$$
\lim _{t \rightarrow \infty} \hat{\alpha}(t)=\alpha \quad \text { and } \quad \lim _{t \rightarrow \infty} \hat{\beta}(t)=\beta
$$




\section{Results and discussion}

In this section, we perform numerical simulations of system (1), based on the results previously obtained. In order to compare the predictions with numerical results, the governing equations of the model (1), were solved numerically using MATLAB [24].

As was obtained in Section 2, the critical (fixed) point of Sprott J system is $E_{0}(0,0,0)$. According to [3], the system (1) is chaotic for $\alpha=2$ and $\beta=2$ with maximal Lyapunov exponent equal to 0.076. Critical point becomes unstable for those values of the parameters, as is observed in equation (2). Numerical simulations were performed with such parameter choice that makes (1) chaotic. The phase portrait of the original chaotic system (1) is shown in Fig. 1. To stabilize the chaotic system at $E_{0}$, adaptive control is introduced along with parameter update laws for the unknown parameters $\alpha$

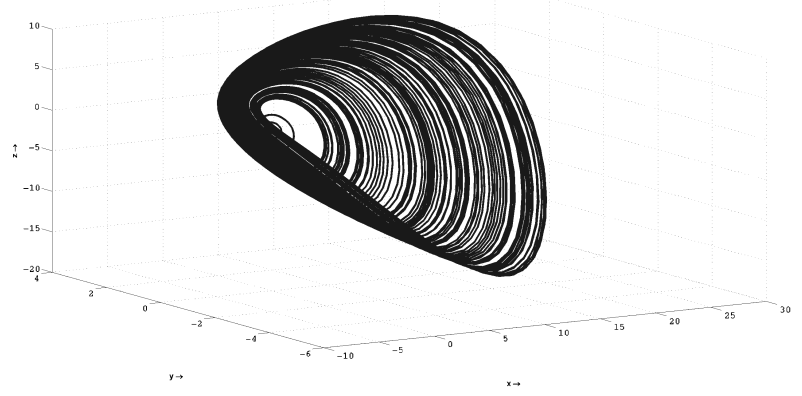

Fig. 1. Phase portrait of Sprott J chaotic system with parameter values $\alpha=2$ and $\beta=2$

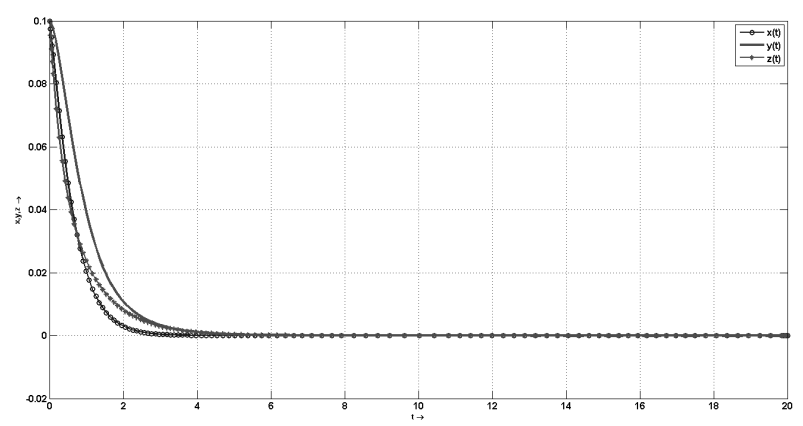

Fig. 2. Adaptive control: Time evolution of the state variables showing convergence to $E_{0}(0,0,0)$ 


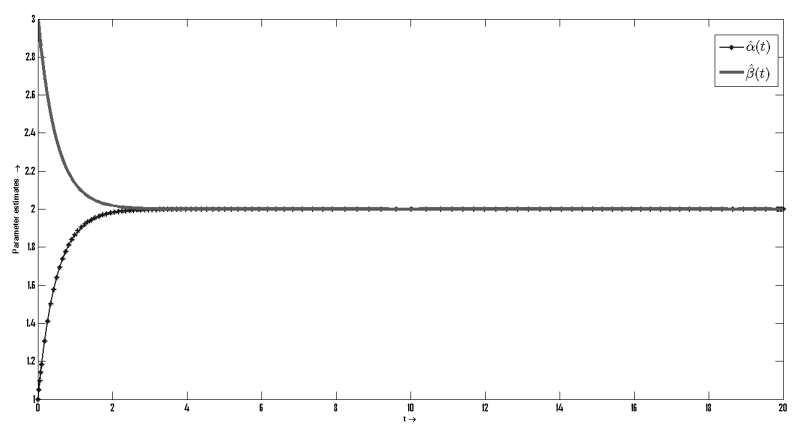

Fig. 3. Adaptive control: Time evolution of the estimates of the system parameters

and $\beta$, as in Section 3. For simplicity of simulation, all the $k_{i}$ are treated as equal, denoted by $k$. The simulation results for adaptive control is given in Figs 2 and 3. The conclusion of the Theorem 2 in Section 3 is supported by the observations in Figs 2 and 3. Figures 2 and 3 are drawn with the initial condition of the state variables $\left(x_{0}, y_{0}, z_{0}\right)=(0.1,0.1,0.1)$ and the parameter estimates $\hat{\alpha}(0)=1, \hat{\beta}(0)=3$ and $k=2$. Figure 2 shows the convergence of the system states to $E_{0}$ and Fig. 3 shows how the estimators asymptotically reach the exact value of the system parameters.

In conclusion, numerical simulations for adaptive synchronization of identical Sprott J system are presented in Figs 4 and 5. The parameters values of the Sprott J system are taken as $\alpha=2, \beta=2$ and the positive constants $k_{i}$ $(i=1,2,3)$ are chosen to be equal, that is $k$. For simulation work, $k$ is chosen to be 5 . The initial conditions imposed on the system for numerical simulation, here are $X(0)=(0.1,0.1,0.1), \hat{X}(0)=(0.2,0,-0.1)$ and $\hat{\alpha}(0)=1, \hat{\beta}(0)=0$.

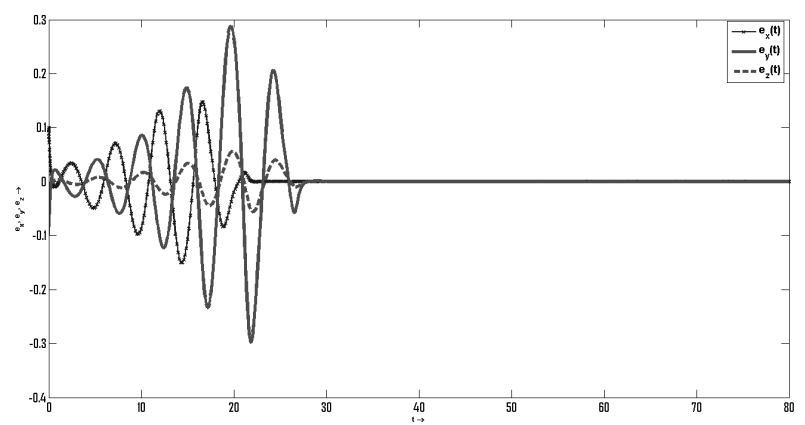

Fig. 4. Convergence of the error between drive and response systems to zero in adaptive synchronization 


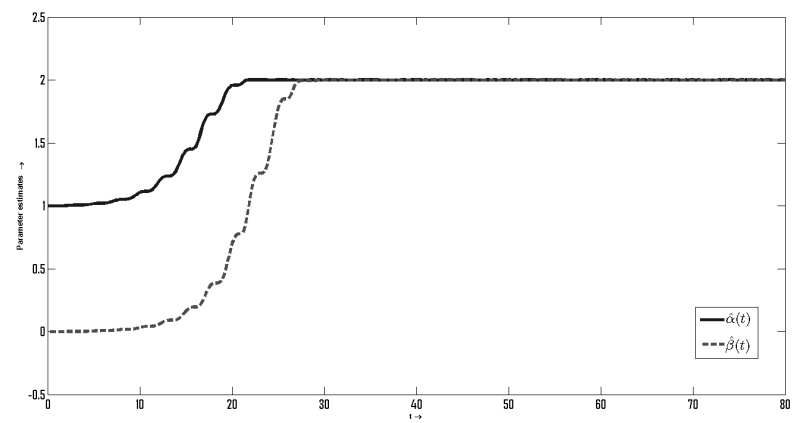

Fig. 5. Adaptive synchronization: Convergence of the system parameter estimators to the actual parameter values $\alpha=2$ and $\beta=2$

The exponential decay of synchronization errors with time is given in Fig. 4. Figure 5 depicts the asymptotic convergence of the parameter estimators to the exact system parameter values. In spite of random choices of initial values of the parameter estimators, they ultimately converge to the actual system parameters. This is a significant achievement and verifies the claims put forth in the paper.

Figures 6 and 7 compare the performance of the control and the synchronization schemes for different values of the control and the coupling parameters, respectively. In order to make a numerical comparison in terms of the control /coupling parameters, we choose all the control/coupling parameters to be equal, that is $k_{i}=k$. Since the convergence discussed in the theory is asymptotic, thus for numerical computation, we have to suitably define a notion determines, whether the system has been controlled or synchronized at

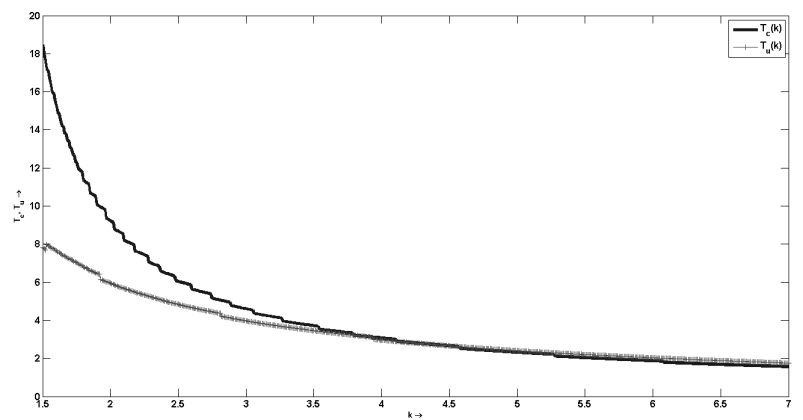

Fig. 6. Performance analysis of the control scheme: Comparison of stabilizaton time $\left(T_{c}\right)$ and parameter estimation time $\left(T_{u}\right)$ for different control parameter values $(k)$ 


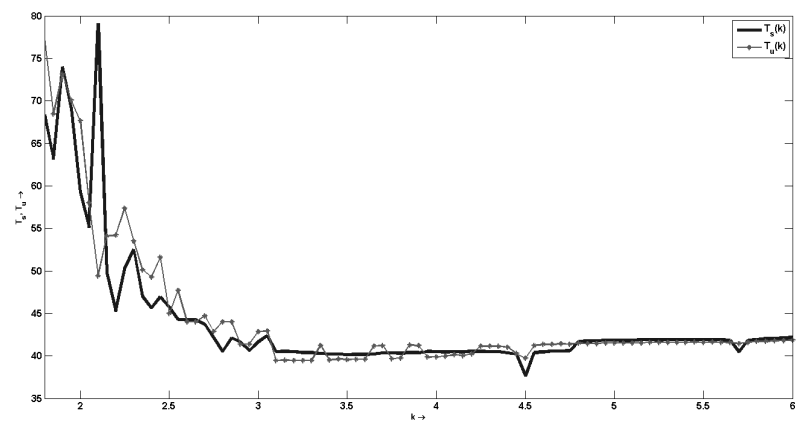

Fig. 7. Pefrormance analysis of the synchronization scheme: Comparison of synchronization time $\left(T_{s}\right)$ and parameter estimation time $\left(T_{u}\right)$ for different values of the coupling parameter $(k)$

a particular point of time. Let $\varepsilon$ be a fixed positive real number and let $\|\cdot\|$ denote the usual Euclidean norm in $\mathbb{R}^{n}$. We consider, that a system $X$ has been controlled if there exists $t_{0}$ such that $\|X\|<\varepsilon$ for all $t>t_{0}$. A pair of systems $(X, Y)$ is synchronized, if there exists $t_{0}$ such that $\|X-Y\|<\varepsilon$ for all $t>t_{0}$. Choosing $\varepsilon$ sufficiently small, this computational approximation provides a fair estimate of the asymptotic convergence. Now, we are in a position to compare the performance of the control and the synchronization schemes, depending on how fast they converge to the required targets.

Let $k$ be the control parameter. Given any $\varepsilon>0$, let us define the following quantities : Stabilization time $T_{c}(k, \varepsilon)=\inf \left\{t_{0} \in \mathbb{R}:\|X(t)\|<\right.$ $\left.\varepsilon, \forall t \geq t_{0}\right\}$ and parameter estimation time $T_{u}(k, \varepsilon)=\inf \left\{t_{0} \in \mathbb{R}:\|\eta(t)\|<\right.$ $\left.\varepsilon, \forall t \geq t_{0}\right\}$, where $\eta(t)=(\hat{\alpha}(t)-\alpha, \hat{\beta}(t)-\beta)$.

Considering $k$ as the coupling parameter, we can further define the quantity:

Synchronization time $T_{s}(k, \varepsilon)=\inf \left\{t_{0} \in \mathbb{R}:\|X(t)-Y(t)\|<\varepsilon, \forall t \geq\right.$ $\left.t_{0}\right\}$.

Here also, we can have the parameter estimation time exactly as defined above.

We fix $\varepsilon$ for our computational study. Figures 6 and 7 are generated by fixing $\varepsilon=10^{-8}$. Variation in the values of $k$ leads to variation of the quantities $T_{c}, T_{s}$ and $T_{u}$, which have been studied graphically in Figs 6 and 7. It is quite easy to observe, that smaller values of the three aforementioned quantities imply better performance of the system, as it achieves its target faster. Thus, Figs 6 and 7 serve the purpose of performance analysis of the proposed control and synchronization schemes with respect to the control and 
the coupling parameter $k$ involved. Figure 6 shows that both $T_{c}$ and $T_{u}$ decrease exponentially as $k$ increases. This conforms with the theoretical result. The control system was theoretically shown to converge exponentially to $E_{0}$ with $k$ determining the rate of convergence. Figure 7 shows the variation of $T_{s}$ and $T_{u}$ with $k$. If one observes the theory, the convergence of errors to zero was not exactly exponential. Hence, the graph doesn't show exponential decrease in synchronization time with increase in $k$. In fact, the graph doesn't even show a very regular pattern, particularly for the low values of $k$. However, for sufficiently large values of $k$, the time of synchronization and the parameter estimation falls off quickly and saturates at a value of about 40 with minor variations.

\section{Conclusion}

In this paper we have analyzed a 3-dimensional chaotic Sprott J system. The focus has been on adaptive control and synchronization. In contrast to many traditional papers in the literature, the result of globally stable synchronization has been established with much weaker restrictions on the derivatives of the Lyapunov function. This has been achieved by using LaSalle invariance principle in place of the more restrictive Lyapunov stability theorems. The numerical results in Section 5 were performed to illustrate the validity and effectiveness of the proposed adaptive control and synchronization scheme in the context of the Sprott J system.

\section{REFERENCES}

[1] Alligood, K. T., T. Sauer, J. A. Yorke. Chaos: An Introduction to Dynamical Systems, New York, Springer, 1997.

[2] Lorenz, E. N. Deterministic Non-periodic Flow. J. Atmos. Sci., 20 (1963), $130-141$.

[3] Sprott, J. C. Some Simple Chaotic Flow. Phys. Rev. E, 50 (1994), No. 2, R647-R650.

[4] Sprott, J. C. Simplest Dissipative Chaotic Flow. Physics Letters A, 228 (1997), 271-274.

[5] Panchev, S. Analytical Properties of the Sprott's Chaotic Flows. Chaos, Solitons and Fractals, 21 (2004), 721-728.

[6] Eichhorn, R., S. Linz, P. Hanggi. Transformations of Nonlinear Dynamical Systems to Jerky Motion and its Application to Minimal Chaotic Flows. Physical Review E, 58 (1998), No. 6, 7151-7164. 
[7] Lerescu, A., N. Constandache, S. Oancea, I. Grosu. Collection of Masterslave Synchronized Chaotic Systems. Chaos, Solitons and Fractals, 22 (2004), 599-604.

[8] Vaidyanathan, S. Adaptive Control and Synchronization of the Uncertain Sprott J System. Int. J. of Mathematics and Scientific Computing, 1 (2011), No. 1, 14-18.

[9] Otт, E., J. C. Sommerer. Blowout Bifurcations: The Occurrence of Riddled Basins and on-off Intermittency. Phys. Lett. A., 188 (1994), 39.

[10] Otт, E., J. C. Sommerer, et al. Scaling Behaviour of Chaotic Systems with Riddled Basins. Phys. Rev. Letters., 71 (1993), 4134.

[11] Ott, E., C. Grebogi, J. Yorke. Controlling Chaos. Phys. Rev Letters, 64 (1990), 1196-1199.

[12] Islam, N., H. P. Mazumdar, A. Das. On the Stability and Control of the Schimizu-Morioka System of Dynamical Equations. Diff. Geo. Dyn. Sys., 11 (2009), 135-143.

[13] Yaseen, M. T. Adaptive Control and Synchronization of a Modified Chua's Circuit System. Applied Mathematics and Computation, 135 (2003), 113-128.

[14] Pikovsky, A., M. Rosenblum, J. Kurths. Synchronization. A Universal Concept in Nonlinear Sciences, Cambridge, Unv. Press, 2001.

[15] Bolotin, Y., A. Tur, V. Yanovsky. Chaos: Concepts, Control and Constructive Use, Berlin, Springer, 2009.

[16] Ge, S. S., C. Wang, T. H. Lee. Adaptive Backstepping Control of a Class of Chaotic Systems. International J. Bifurcation and Chaos, 10 (2000), No. 5, 1149-1156.

[17] Wang, X., L. Tian, L. Yu. Adaptive Control and Slow Manifold Analysis of a New Chaotic System. International J. Nonlinear Science, 21 (2006), 43-49.

[18] Poria, S., A. Taria (Poria). Adaptive Synchronization of Two Coupled Chaotic Neuronal Systems. Rev. Bull. Cal. Math Soc., 15 (2007), No. 1, 53-60.

[19] Feng, J., S. Chen, C. Wang. Adaptive Synchronization of Uncertain Hyperchaotic Systems Based on Parameter Identification. Chaos, Soltions and Fractals, 26 (2005), 1163-1169.

[20] Sun, M., L. Tian, S. Jiang, J. Xun. Feedback Control and Adaptive Control of the Energy Resource Chaotic System. Chaos, Solitons and Fractals, 32 (2007), $168-180$.

[21] Astrom, K. J., B. Wittenmark. Adaptive Control, 2nd edition, USA, New Jersey, Prentice Hall, 1994.

[22] Pecora, L. M., T. L. Caroll. Synchronization in Chaotic Systems. Phys. Rev. Letters, 64 (1990), 821-824.

[23] Feki, M. An Adaptive Chaos Synchronization Scheme Applied to Secure Communication. Chaos, Solitons and Fractals, 18 (2003), 141-148.

[24] MATLAB, The MathWorks, Inc., 2010, http://www.mathworks.com/. 